\title{
ACCURATE PREDICTOR-CORRECTOR SKIP ENTRY GUIDANCE FOR LOW LIFT-TO-DRAG RATIO SPACECRAFT
}

\author{
Y. Enmi ${ }^{1,2}, W \cdot \operatorname{Qian}^{1,2}$, K. He ${ }^{1,2}$, and D. $\mathbf{D i}^{1,2}$ \\ ${ }^{1}$ State Key Laboratory of Aerodynamics \\ Mianyang Sichuan 621000, China \\ ${ }^{2}$ China Aerodynamics Research and Development Center \\ Mianyang Sichuan 621000, China
}

\begin{abstract}
This paper develops numerical predictor-corrector skip entry guidance for vehicles with low lift-to-drag $L / D$ ratio during the skip entry phase of a Moon return mission. The guidance method is composed of two parts: trajectory planning before entry and closed-loop guidance during skip entry. The result of trajectory planning before entry is able to present an initial value for predictor-corrector algorithm in closed-loop guidance for fast convergence. The magnitude of bank angle, which is parameterized as a linear function of the range-to-go, is modulated to satisfy the downrange requirements. The sign of the bank angle is determined by the bank-reversal logic. The predictor-corrector algorithm repeatedly applied onboard in each guidance cycle to realize closed-loop guidance in the skip entry phase. The effectivity of the proposed guidance is validated by simulations in nominal conditions, including skip entry, loft entry, and direct entry, as well as simulations in dispersion conditions considering the combination disturbance of the entry interface, the aerodynamic coefficients, the air density, and the mass of the vehicle.
\end{abstract}

\section{INTRODUCTION}

Recent Space exploration missions, such as ORION mission from NASA, require an entry guidance approach can realize a wide range of entry trajectories with a high landing accuracy. Although the Apollo skip reentry guidance has been successfully applied in 1960s, the various approximations and linearization assumptions in the guidance have been found to limit the accuracy when the downrange and crossrange are relative long. With the development of flight

(C) The Authors, published by EDP Sciences. This is an open access article distributed under the terms of the Creative Commons Attribution License 4.0 (http://creativecommons.org/licenses/by/4.0/). 
computers, onboard calculation of a trajectory has become available. Some numerical predictor-corrector guidance algorithms appeared in literature research recently [1-6].These methods are not limited by the validity of the simplifications, approximations, and empirical assumptions necessary for any analytic treatment. Therefore, they hold greater potential to be more accurate and adaptive. The representative of them are the two Orion skip entry guidance: the Numerical Skip Entry Guidance(NSEG) developed at NASA/JSC and PreGuid developed at the Charles Stark Draper Laboratory $[1,2]$. Both algorithms use a numerical predictor-corrector for the skip phase coupled with the Apollo final phase entry guidance. Putnam et al. also presented a skip entry guidance by introducing a numerical predictor-corrector phase during the atmospheric skip portion with an Apollo guidance baseline algorithm [3]. Vernis et al. reported a numerical predictor-corrector entry guidance that had been designed within the frame of the ESA-funded 'Robust skip Entry' program [6]. A succession of three constant bank angle phases with three roll reversals for the Moon return mode of this guidance is required. Therefore the correction of the guidance algorithm included two parts: the cross-range correction and the downrange correction which may need high performance of onboard processor. A full numerical predictorcorrector method for trajectory planning and closed-loop guidance of skip entry was put forward by Christopher and Ping. The problem is formulated as a nonlinear uni-variate root-finding problem in downrange control guidance and bank reversal problem in lateral guidance $[4,5]$.

In this paper, a numerical predictor-corrector approach motivated by similar principles of Christopher and Ping is developed for the skip entry problem which can cover the cases of direct entry, loft entry, and skip entry cases in lunar return mission.

\section{SKIP ENTRY GUIDANCE PROBLEM DESCRIPTION}

The general purpose of reentry guidance is to guide the reentry vehicle from entry interface (EI) to the specified landing site in the disturbance circumstance of the Earth's atmosphere. The typical skip reentry trajectory is illustrated in Fig. 1. For the capsule type reentry vehicle, the guidance algorithms modulate the bank angle to achieve the specified terminal constraints land point while using a trim angle-of-attack. So, the magnitude and the sign of the bank angle are determined by the guidance algorithm which will be described in the next section.

The flight of a return capsule is subject to the vehicle dynamics described by the following dimensionless equations of three-dimensional motion for a point mass about a rotating circular Earth: 


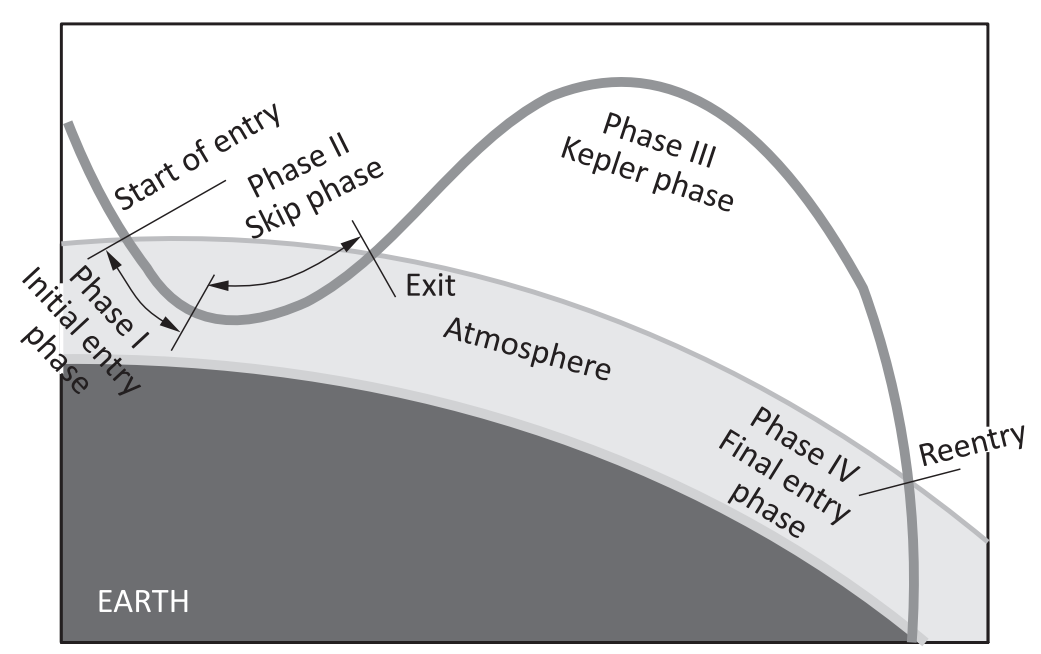

Figure 1 Typical skip entry trajectory

$$
\begin{aligned}
& \frac{d r}{d \tau}= V \sin \gamma^{*} ; \\
& \frac{d \theta}{d \tau}= \frac{V \cos \gamma \sin \psi}{r \cos \varphi} ; \\
& \frac{d \varphi}{d \tau}= \frac{V \cos \gamma \cos \psi}{r} ; \\
& \frac{d V}{d \tau}=-\bar{D}-\frac{\sin \gamma}{r^{2}}+\Omega^{2} r \cos \varphi(\sin \gamma \cos \varphi-\cos \gamma \sin \varphi \cos \psi) ; \\
& \frac{d \gamma}{d \tau}= \frac{1}{V}\left[\bar{L} \cos \sigma+\frac{\cos \gamma}{r}\left(V^{2}-\frac{1}{r}\right)\right. \\
&\left.+2 \Omega V \cos \varphi \sin \psi+\Omega^{2} r \cos \varphi(\cos \gamma \cos \varphi+\sin \gamma \cos \psi \sin \varphi)\right] \\
& \frac{d \psi}{d \tau}= \frac{1}{V}\left[\frac{\bar{L} \sin \sigma}{\cos \gamma}+\frac{V^{2}}{r} \cos \gamma \sin \psi \tan \varphi-2 \Omega V(\tan \gamma \cos \varphi \cos \psi-\sin \varphi)\right. \\
&\left.\quad+\frac{\Omega^{2} r}{\cos \gamma} \sin \varphi \cos \varphi \sin \psi\right]
\end{aligned}
$$

Here, $r$ is the radial distance from the center of the Earth to the vehicle, normalized by the average radius of the Earth $R_{0}=6378 \mathrm{~km}$. The Earth relative velocity is $V$, normalized by $V_{c}=\sqrt{g_{0} R_{0}}$ where $g_{0}=9.81 \mathrm{~m} / \mathrm{s}^{2}$. The longitude, latitude, flight path angle (FPA), and velocity azimuth angle are $\theta, \varphi, \gamma$, and $\psi$, respectively. The heading angle $\psi$ is measured from the north in a clockwise 
direction. Derivatives of these variables are taken with respect to the dimensionless time $\tau=t / \sqrt{R_{0} / g_{0}}$. The dimensionless angle velocity $\Omega$ is the Earth self-rotation rate normalized by $\sqrt{g_{0} / R_{0}}$. The bank angle is $\sigma$ acting as one of the main control variables, $\bar{L}$ and $\bar{D}$ represent the nondimensional lift and drag accelerations, in terms $g_{0}$, and can be calculated from

$$
\bar{L}=\frac{\rho\left(V V_{c}\right)^{2} S C_{L}}{2 m g_{0}} ; \quad \bar{D}=\frac{\rho\left(V V_{c}\right)^{2} S C_{D}}{2 m g_{0}} .
$$

\section{PROPOSED SKIP ENTRY GUIDANCE APPROACH}

\subsection{The Outline of the Guidance Logic}

There are four phases in this guidance law both in trajectory planning and closedloop guidance (see Fig. 1):

Phase I: Initial entry phase which is from the EI to the skip altitude;

Phase II: Skip phase which is from the end of phase I to the atmosphere exit point;

Phase III: Kepler phase which is from the exit point to the reentry point; and

Phase IV: Final entry phase which is from the reentry point to the parachute deploying point.

The basic scheme of the proposed guidance is a numerical predictor-corrector algorithm in which the equations of motion are integrated with a fourth-order Runge-Kutta method adopted by the onboard predictor. Further more, there are different forms of predictor-corrector algorithms used in the different phases of skip guidance process in order to deal with the challenges in the lunar return cases including the initial velocity of about $11 \mathrm{~km} / \mathrm{s}$, varied entry range requirements from 2500 to $10000 \mathrm{~km}$, and the possibility of bouncing off or diving steeply into the atmosphere in the disturbance circumstances.

The guidance phase transition logic and the algorithm description of each phase are illustrated in Fig. 2. The initial entry phase begins at the entry interface. The guidance algorithm regulates the magnitude of $\sigma_{d}$ which is a design parameter of the bank angle (see Fig. 2 and Eq. (8) below) to make the final range-to-go less than the given final phase range $\left(F_{\text {stogo }}\right)$ until the altitude below $80 \mathrm{~km}$. The guidance algorithm, in this phase is benefit to avoid the case of bouncing off the atmosphere and too much computational burden if using a high accuracy predictor-corrector algorithm which should propagate the nearly whole skip reentry trajectory in every prediction cycle. Then, the guidance logic is transferred to the skip phase in which the magnitude of $\sigma_{d}$ is iterated by a scant 


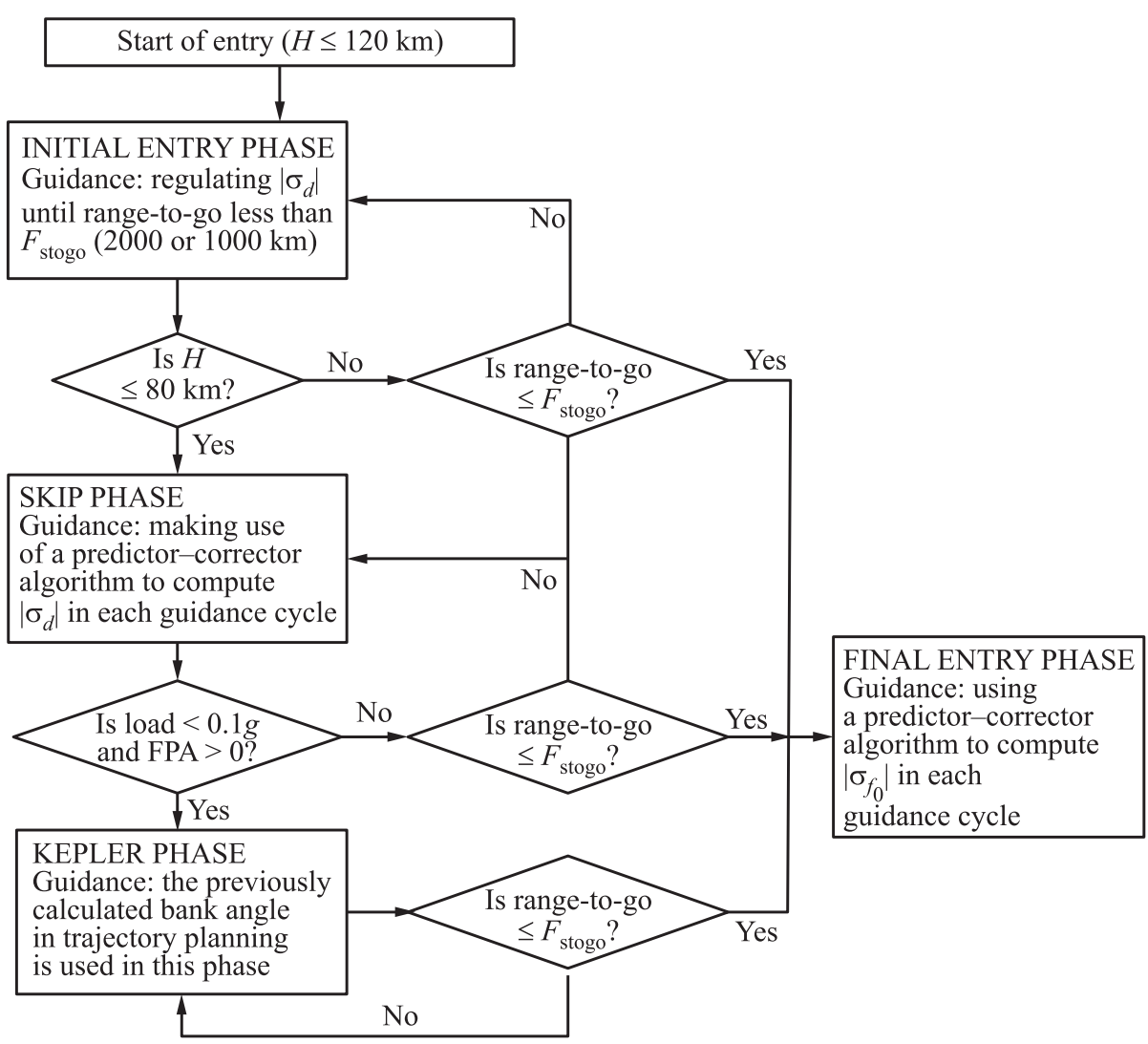

Figure 2 Guidance phase transition logic and algorithm description ( $H$ denotes the altitude, FPA denotes the flight path angle, and $F_{\text {stogo }}$ denotes the given final phase range)

method to satisfy the range requirement with a relatively high accuracy in every guidance cycle. If the $g$-load under the value 0.1 , the guidance automatically transit to the Kepler phase. In this phase, the previous designed bank angle $\left|\sigma_{d}\right|$ is stained and the current magnitude of bank angle is computed by Eq. (8). When the $g$-load exceeds the value 0.1 again or the range-to-go is less than the given final phase range, the guidance law is switched to the final phase guidance in which a new model of bank angle (see Fig. 4 below) and equations of motion (see Eqs. (13) below) for prediction are adopted. Then, the initial bank angle of the final phase $\sigma_{f_{0}}$ is the design variable of the final predictor-corrector algorithm.

The above transition logic of guidance is for the normal skip entry case. There are two other branches you can see in Fig. 2 which is for the loft entry and 
director entry cases. After the initial entry phase, if the range-to-go is already less than the given final phase range, the guidance law is directly transferred to phase IV. That is a direct entry case. Otherwise, if the range-to-go is less than the $F_{\text {stogo }}$ in the process of skip phase, it also transits to phase IV. This case is called loft entry. Then, the guidance logic presented here is adaptive to both the long and the short range cases which are required by the near-future Moon return projects.

\subsection{Trajectory Planning Algorithm}

In trajectory planning, the magnitude of the bank angle is parameterized as a linear function of the range-to-go until a specified threshold $s_{\text {to-go }}^{f_{0}}$, which is the start of the final entry phase and the range of this phase depends on the total range. The choice of distance of final entry phase $\left(F_{\text {stogo }}\right)$ is such that it represents typical downrange at the initiation of the final phase guidance. According to simulation experience, it is taken the value of $2000 \mathrm{~km}$ when the total range is longer than $5000 \mathrm{~km}$; otherwise, it is taken as $1000 \mathrm{~km}$. These values do not vary widely for low $L / D$ reentry vehicles. When the current rangeto-go is less than $s_{\text {to-go }}^{f_{0}}$, a constant bank-angle magnitude of $\sigma_{f}$ is used. Figure 3 shows the bank angle profile. Then, the function of the bank angle magnitude $|\sigma|$ is described:

$$
|\sigma|= \begin{cases}\left|\sigma_{d}\right|+\left(\left|\sigma_{0}\right|-\left|\sigma_{d}\right|\right) \frac{s_{\text {to-go }}-s_{\text {to-go }}^{d}}{s_{\text {to-go }}^{0}-s_{\text {to-go }}^{d}}, & s_{\text {to-go }}^{d} \leq s_{\text {to-go }} \leq s_{\text {to-go }}^{0} \\ \left|\sigma_{f}\right|+\left(\left|\sigma_{d}\right|-\left|\sigma_{f}\right|\right) \frac{s_{\text {to-go }}-s_{\text {to-go }}^{f_{0}}}{s_{\text {to-go }}^{d}-s_{\text {to-go }}^{f_{0}}}, & s_{\text {to-go }}^{f_{0}} \leq s_{\text {to-go }} \leq s_{\text {to-go }}^{d} \\ \left|\sigma_{f}\right|, & s_{\text {to-go }}<s_{\text {to-go }}^{f_{0}}\end{cases}
$$

where

$$
s_{\text {to-go }}^{d}=s_{\text {to-go }}^{f_{0}}+\frac{3}{4}\left(s_{\text {to-go }}^{0}-s_{\text {to-go }}^{f_{0}}\right) .
$$

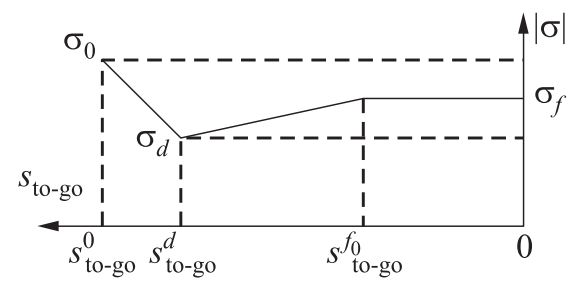

Figure 3 Bank-angle parameterization model in trajectory planning
The trajectory planning algorithm tries to find the bank angle $\left|\sigma_{d}\right|$ to satisfy the total range requirements. Through the integration of the equations of motion (1)-(6) with $|\sigma|$ given by Eqs. (8), (9), and the bank reversal logic that will be given in subsection 3.4, the search for $\left|\sigma_{d}\right|$ become a one root searching 
problem: at the current state, find the $\left|\sigma_{d}\right|$ that will satisfy the total range requirements,

$$
f\left(\left|\sigma_{d}\right|\right)=s_{\mathrm{to}-\mathrm{go}_{f}}\left(\left|\sigma_{d}\right|\right)=0 .
$$

The integration of the equations of motion is end up at the parachute deploying altitude $10 \mathrm{~km}$. The final range-to-go when arrive at this altitude will be positive if the reentry vehicle undershoots and negative if there is overshoot. Here, a relatively simple secant method is utilized to iteratively solve Eq. (10) for saving computational resource onboard. The iteration equation is:

$$
\left|\sigma_{d}\right|^{i+1}=\left|\sigma_{d}\right|^{i}-\left(\frac{\left|\sigma_{d}\right|^{i}-\left|\sigma_{d}\right|^{i-1}}{s_{f}^{i}-s_{f}^{i-1}}\right) s_{f}^{i} .
$$

In this equation, where $s_{f}^{i}$ denotes the final range-to-go using the value of $\left|\sigma_{d}\right|^{i}$ to propagate the equation of motion. When the iteration succeeds, a feasible skip entry trajectory can be obtained and the value of $\left|\sigma_{d}\right|$ also can be used as an initial value in the closed-loop guidance.

\subsection{Closed-Loop Guidance}

To cope with the uncertainties of the varied range requirement, the closed-loop guidance is carried out after trajectory planning at the EI which repeatedly generates a feasible skip trajectory using the current state as an initial state in each guidance cycle.

The closed-loop guidance imposes the same algorithm to modulate the bank angle until the end of Kepler phase. In the final entry phase, the longitude equations of motion and a new bank angle function are implemented in the closed-loop guidance to improve the final accuracy and the convergence rate. The bank angle function is a linear function of the dimensionless energy $e$ which is defined as [7]

$$
e=\frac{1}{r}-\frac{V^{2}}{2}
$$

Figure 4 shows the bank angle profile of phase IV in closed-loop guidance. Therefore, the bank angle magnitude $|\sigma|$ at any energy $e$ in final entry phase is:

$$
|\sigma|=\left|\sigma_{f_{0}}\right|+\left(\left|\sigma_{f}\right|-\left|\sigma_{f_{0}}\right|\right) \frac{e-e_{f_{0}}}{e_{f}-e_{f_{0}}}
$$

where $e_{f_{0}}$ is the current dimensionless energy; and $e_{f}$ is the specified dimensionless energy in parachute deployment condition with a velocity of $200 \mathrm{~m} / \mathrm{s}$ and an altitude of $10 \mathrm{~km}[4]$. 
With the assumption of great circle, the

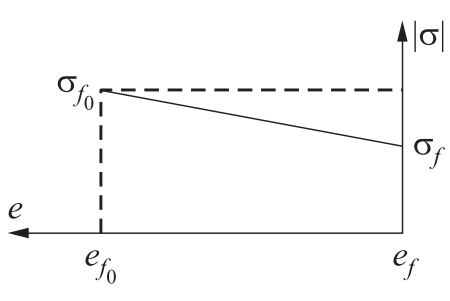

Figure 4 Bank-angle parameterization model in the final phase in closed-loop guidance range-to-go $s$ satisfies the following differential equation:

$$
\dot{s}=-\frac{V \cos \gamma}{r} .
$$

One can see that from the equations of motion, the longitude motion is decoupled from the lateral motion variables when the Earth's rotation effects are ignored. Therefore, the longitude motion with Eq. (12) can be described as follows:

$$
\begin{aligned}
r & =V \sin \gamma \\
\dot{V} & =-\bar{D}-\frac{\sin \gamma}{r^{2}} \\
\dot{\gamma} & =\frac{1}{V}\left[L \cos \sigma+\left(V^{2}-\frac{1}{r}\right) \frac{\cos \gamma}{r}\right] .
\end{aligned}
$$

Then, eliminating the equation for $V$ by using the dimensionless energy (Eq. (11)) as the independent variable, one can deduce the dimensionless longitude equations [7]:

$$
\left.\begin{array}{l}
\frac{d s}{d e}=-\frac{\cos \gamma}{r \bar{D}} \\
\frac{d r}{d e}=\frac{\sin \gamma}{\bar{D}} \\
\frac{d \gamma}{d e}=\frac{1}{\bar{D} V}\left[L \cos \sigma+\left(V^{2}-\frac{1}{r}\right) \frac{\cos \gamma}{r}\right]
\end{array}\right\}
$$

where $V=\sqrt{2(1 / r-e)}$. Set the initial values of the state variables to be the current condition. For a given magnitude profile of the bank angle $|\sigma|$, the integration of Eqs. (13) from the current condition to the specified final energy gives the final range-to-go $s_{f}\left(e_{f}\right)$. Then, the predictor-corrector guidance problem in phase IV can be formulated as: the initial magnitude of bank angle in final phase $\left|\sigma_{f_{0}}\right|$ is to be found to satisfy the final range requirements. Through the integration of the equations of motion (13), the final range-to-go can be regarded as a function of $\left|\sigma_{f_{0}}\right|$. A scant method is still adopted to solve the one-parameter problem, the iterate equations on $\left|\sigma_{f_{0}}\right|$ are given by:

$$
\left|\sigma_{f_{0}}\right|^{(i+1)}=\left|\sigma_{f_{0}}\right|^{(i)}-\frac{s_{f}\left(\left|\sigma_{f_{0}}\right|^{(i)}\right)}{\partial s_{f}\left(\left|\sigma_{f_{0}}\right|^{(i)}\right) / \partial \sigma}
$$

where the partial derivative $\partial s_{f}\left(\left|\sigma_{f_{0}}\right|^{(i)}\right) / \partial \sigma$ is obtained by a finite-difference approximation. 


\subsection{Lateral Guidance Logic}

The lateral guidance logic utilizes a bank reversal logic as usual in reentry guidance law with a cross range variable defined as

$$
\chi=\sin ^{-1}\left[\sin \left(s_{\text {to-go }}\right) \sin \left(\psi-\Psi_{\text {LoS }}\right)\right] .
$$

Here, $\Psi_{\text {LoS }}$ is the line-of-sight azimuth angle along a great circle to the landing site defined by

$$
\Psi_{\mathrm{LoS}}=\arctan \left[\frac{\sin \left(\theta_{f T}-\theta\right)}{\cos \varphi \tan \varphi_{f T}-\sin \varphi \cos \left(\theta_{f T}-\theta\right)}\right]
$$

where $\theta_{f T}$ and $\varphi_{f T}$ are the target parachute deployment longitude and latitude, respectively. Bank reversal logic is employed to determine the sign of bank angle for trajectory prediction both in trajectory planning and in closed-loop guidance. Then, the sign of bank angle is reversed whenever the cross range exceeds the velocity-dependent dead band [8] whose width is defined by

$$
y=C_{1} V+C_{0}
$$

where $C_{1}$ and $C_{0}$ are two constants. This Apollo-like lateral logic regulates the cross range to ensure a flight direction toward the landing site. The lifting capability, size, and weight of a vehicle determine how quickly it can change its flight direction and unnecessary bank reversals could prematurely deplete the propellant of the reaction control system. Therefore, the constants $C_{1}$ and $C_{0}$ may need to be tuned for different vehicles for a good balance between crossrange regulation tightness and reasonable number of bank reversals. In this paper, the following values have been used: $C_{0}=1.7 \cdot 10^{-5}$ and $C_{1}=5.2 \cdot 10^{-3}$.

\subsection{Critical Issues in Guidance Scheme}

Several critical issues in guidance scheme which can improve the adaptivity and robustness implemented in this paper are as follows.

\section{A. A parameterized aerodynamic model for prediction}

A parameterized aerodynamic model is constructed to get the trimmed aerodynamic coefficients instead of the general looking table method to mostly reduce the prediction computation time. Here, a structured aerodynamic model for parameter identification of a reentry experimental vehicle is utilized [9]. The original model is simplified in the trim condition as follows:

$$
\begin{aligned}
C_{D} & =\left(C_{D 0} \cos ^{2} \alpha+C_{D \alpha} \cos \alpha \sin \alpha+C_{D \alpha^{2}} \sin ^{2} \alpha\right) ; \\
D_{L} & =\left(C_{L 0} \cos ^{2} \alpha+C_{L \alpha} \cos \alpha \sin \alpha+C_{L \alpha^{2}} \sin ^{2} \alpha\right) .
\end{aligned}
$$


The model allows one to analytically express each aerodynamic coefficient as a nonlinear function of Mach number and a set of constant aerodynamic parameters. These parameters, $C_{D 0}, C_{D \alpha}, C_{D \alpha^{2}}, C_{L 0}, C_{L \alpha}$, and $C_{L \alpha^{2}}$, are identified by the least square method based on the original aerodynamic data of a low $L / D$ return capsule.

\section{B. A Pre-Guid algorithm in guidance cycle}

In the Moon exploration mission, the vehicle entry into the atmosphere of the Earth with a near second cosmic velocity, the trajectory is most likely to bounce off the atmosphere or dive deeply into the atmosphere in a given bank angle. When the instance happened in prediction process, the range-to-go will be a nonmonotonous function of the iteration parameter. Then, the scant method may fail in solving the root-finding problem. This paper introduces a Pre-Guid algorithm to regulate the predict trajectory in a tolerable range which requires the final range-to-go less than $F_{\text {stogo }}$, which was defined in subsection 3.2, before iteration. This Pre-Guid algorithm decreases the designed bank angle $\left|\sigma_{d}\right|$ when the predict trajectory bounces off the atmosphere or overshoots whereas increased the bank angle $\left|\sigma_{d}\right|$ when the predict trajectory is under shoot. The regulate step of the bank angle $\left|\sigma_{d}\right|$ is varied from $5^{\circ}$ to $0.1^{\circ}$ according to the predicted final range-to-go to insure the fast convergence.

\section{Adaptation of the prediction integration steps and convergence accuracy}

The primary disadvantage of a numerical predictor-corrector algorithm is the onboard computational burden especially in the long range condition [6]. To alleviate the computational burden in prediction, the RK4 (4th-order RungeKutta) prediction step and convergence accuracy is adapted in the different flight phases as described in Table 1.

Table 1 Adaption of the prediction integration steps

\begin{tabular}{lcc}
\hline \multicolumn{1}{c}{ Phase } & $\begin{array}{c}\text { Integration } \\
\text { step, } \\
\mathrm{s}\end{array}$ & $\begin{array}{c}\text { Convergence } \\
\text { accuracy, } \\
\mathrm{km}\end{array}$ \\
\hline Initial entry & 10 & $2000 / 1000$ \\
Skip & 10 & 25 \\
Kepler & 20 & 25 \\
Final entry & 2 & 5 \\
\hline
\end{tabular}




\section{Lift and drag filters}

It is recommended in the relative literature that the appropriate use of estimated information on the aerodynamic and density biases is very beneficial to obtain high accuracy [10-13]. A first-order fading-memory filter is used [4, 14]:

$$
X_{n+1}=X_{n}+(1-\beta)\left(X^{*}-X_{n}\right)
$$

where $X^{*}$ is the current ratio of the measured variable (lift or drag acceleration) to its nominal value based on the nominal model via Eqs. (7), $X_{n}$ is the past filtered ration and $0<\beta<1$ is the gain chosen to emphasize past values over the most recent value. To initialize the filter, the first past filtered ratio $X_{0}=1$. In each guidance cycle, the output of this filter is used to multiply the nominal lift and drag profiles in the integration of the trajectory.

\section{PERFORMANCE OF THE PROPOSED GUIDANCE ALGORITHM}

To evaluate the performance of the proposed reentry guidance algorithm, detailed numerical simulations are carried out with the following conditions: $(i)$ nominal condition in different downrange requirements situation; and (ii) perturbation condition considering the dispersion reentry interface, the disturbance of aerodynamic coefficients, the air density, and the mass of the vehicle.

\subsection{Assumptions and Design Parameters for Simulation Studies}

The assumptions and the main design parameters used for verification of the algorithm are as follows.

A. The guidance algorithm is implemented on a simulation platform of $3 \mathrm{DoF}$ (three degrees-of-freedom) described by equations of motion (1)-(6). Then, the RK4 integral method is used for numerical integration and with a time step of a half second. The guidance cycle is $2 \mathrm{~s}$. That is to say, in every $2 \mathrm{~s}$, the predictor-corrector algorithm is implemented once. The simulation is executed in the software environment of MATLAB.

B. According to the case of the Apollo-type entry vehicle, it was assumed that the initial altitude of the reentry at EI was $400,000 \mathrm{ft}$ which corresponds to $121.92 \mathrm{~km}$. For simplicity, an equatorial orbit with initial position of zero latitude and zero longitude and a typical lunar return velocity of $11,032 \mathrm{~m} / \mathrm{s}$ are chosen. The initial FPA and azimuth angle are $-5.9^{\circ}$ and $90^{\circ}$, respectively.

C. The terminal requirements in range-to-go and altitude are $\leq 5 \mathrm{~km}$ and equal to $10 \mathrm{~km}$, respectively. 


\subsection{Performance in Nominal Condition}

In the normal entry cases, simulation was performed for 5 different target locations with respect entry interface: 10,000, 7,300, $4,600,3,500$, and $2,400 \mathrm{~km}$. The 2,400 -kilometer range was chosen because it was also the approximate minimum range required by Apollo vehicles. Otherwise, the 10,000-kilometer range represents the typical range of a skip entry. The other ranges are between these two values to cover the three types, which are direct entry, loft entry, and skip entry, possibly appeared in lunar return

Table 2 Range requirements and errors in normal condition simulation

\begin{tabular}{ccr}
\hline Case & $\begin{array}{c}\text { Range } \\
\text { requirements, } \\
\mathrm{km}\end{array}$ & $\begin{array}{c}\text { Miss } \\
\text { range, } \\
\mathrm{km}\end{array}$ \\
\hline 1 & 10,000 & -2.10 \\
2 & 7,300 & 0.87 \\
3 & 4,600 & -1.78 \\
4 & 3,500 & -0.46 \\
5 & 2,400 & -2.10 \\
\hline
\end{tabular}
mission.

The main simulation results are listed in Table 2 and Fig. 5. In normal entry condition, all the simulation cases performed here are able to satisfy the accuracy

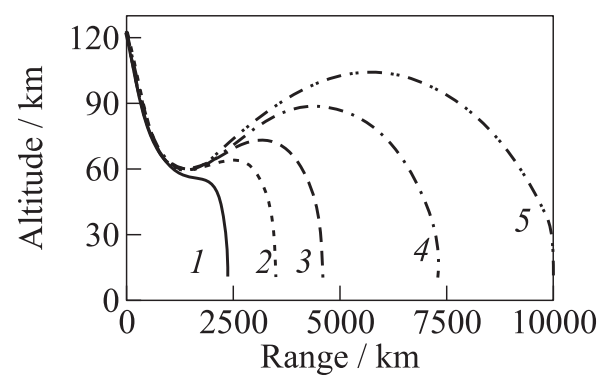

(a)

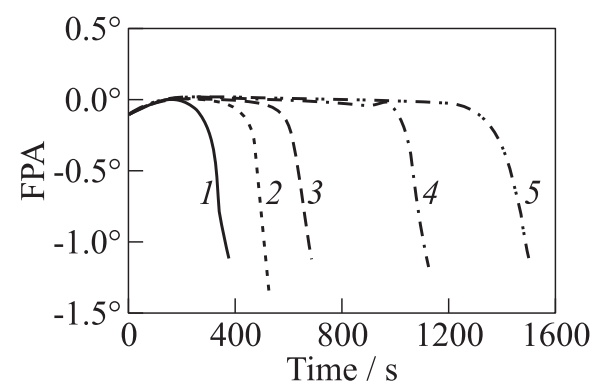

(c)

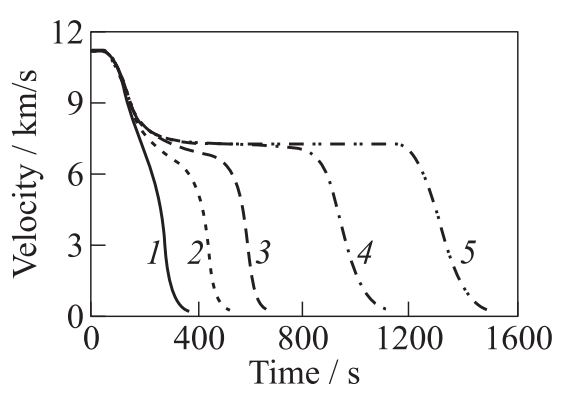

(b)

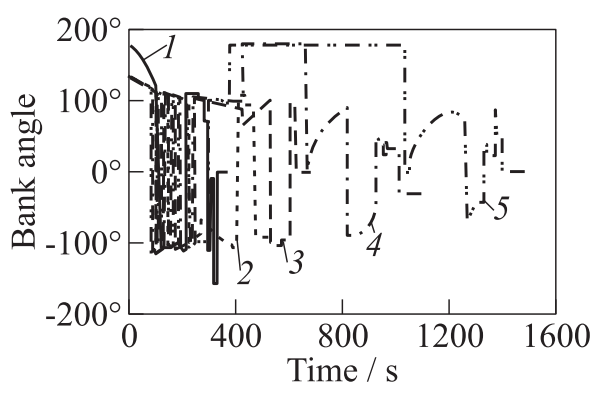

$(d)$

Figure 5 Simulation results in normal entry condition: $(a)$ altitude vs. range; $(b)$ velocity vs. time; $(c)$ FPA vs. time; $(d)$ bank angel vs. time; $1-2,400 \mathrm{~km} ; 2-3,500$; $3-4,600 ; 4-7,300$; and $5-10,000 \mathrm{~km}$ 
requirements (see Table 2). It demonstrates that the guidance transition logic and algorithms described in Fig. 2 are valid for the varied range conditions in lunar return mission. The bank angle, as the control variable in guidance problem, is in the efficient scope and without too much bank reversal. In the guidance cycles, the iterative convergence just needs 3 or 4 steps, and the computational time is less than the guidance cycle which is $2 \mathrm{~s}$ in these cases.

\subsection{Performance Under Disturbed Flight Environment}

To assess the performance of the guidance algorithm under conditions of dispersed reentry interface, the simulations have been carried out with the assumed dispersion on the reentry initial states, the air density, mass of vehicle, and aerodynamic coefficients, as given in Table 3. Each of the dispersion is described by a scalar value which is assumed to be a Gaussian distribution and determined by a mean value $(\mu)$ and a maximum expected perturbation $(3 \sigma)$. Here, the same perturbation values are adopted as reference [2]. For perturbations introduced as a multiplier, the same multiplier is applied during the whole range for one time Monte-Carlo simulation.

Table 3 Parameters used in Monte-Carlo analysis

\begin{tabular}{lclcc}
\hline Parameter & $\begin{array}{c}\text { Mean } \\
\text { value } \mu\end{array}$ & Reason for mean & $\begin{array}{c}3 \sigma \\
\text { deviation }\end{array}$ & Reason for $3 \sigma$ \\
\hline EI velocity & $11,032 \mathrm{~m} / \mathrm{s}$ & $\begin{array}{l}\text { Lunar return } \\
\text { velocity }\end{array}$ & $300 \mathrm{~m} / \mathrm{s}$ & $\begin{array}{l}\text { Based on various } \\
\text { lunar departure } \\
\text { declinations }\end{array}$ \\
\hline EI azimuth & $90^{\circ}$ (East) $)$ & $\begin{array}{l}\text { Equatorial orbit } \\
\text { for simplicity }\end{array}$ & $0.1^{\circ}$ & $\begin{array}{l}\text { Equal to orbital } \\
\text { inclination error [2] }\end{array}$ \\
\hline EI FPA & $-5.9^{\circ}$ & $\begin{array}{l}\text { Analysis by } \\
\text { Draper Laboratory }\end{array}$ & $0.1^{\circ}$ & $\begin{array}{l}\text { Based on 0.5 nmi } \\
\text { vacuum periapse } \\
\text { altitude error [14] }\end{array}$ \\
\hline Vehicle mass & $3200 \mathrm{~kg}$ & $\begin{array}{l}\text { Ordinary CEV-type } \\
\text { vehicle }\end{array}$ & $5 \%$ & \begin{tabular}{c} 
Design uncertainty \\
\hline $\begin{array}{l}\text { Atmospheric } \\
\text { density } \\
\text { multiplier }\end{array}$
\end{tabular} \\
\hline$C_{D}$ multiplier & 1 & $\begin{array}{l}\text { Definition } \\
\text { of multiplier }\end{array}$ & $20 \%(0.2)$ & $\begin{array}{c}\text { Day of flight } \\
\text { uncertainty }\end{array}$ \\
\hline$C_{L}$ multiplier & 1 & $\begin{array}{l}\text { Definition } \\
\text { of multiplier }\end{array}$ & $20 \%(0.2)$ & $\begin{array}{l}\text { Based on CFD } \\
\text { analysis and test } \\
\text { flight calibration }\end{array}$ \\
\hline
\end{tabular}




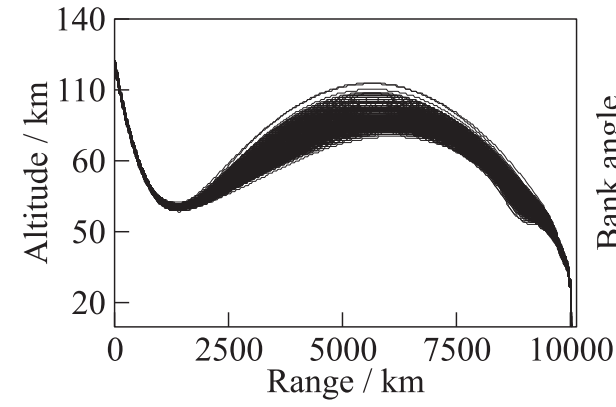

(a)

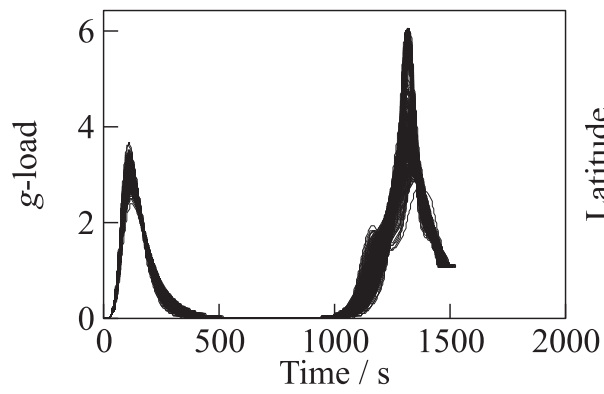

(c)

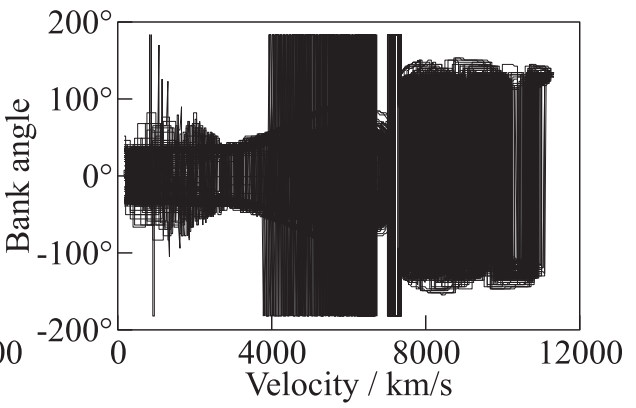

(b)

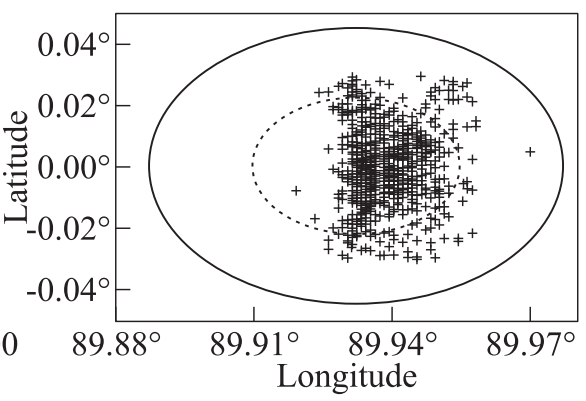

(d)

Figure 6 Monte-Carlo results for 1000 dispersed trajectories: (a) altitude vs. range; (b) bank angel vs. velocity; (c) g-load vs. time; and (d) landing error distribution (crosses inside the inner dotted curve refer to $2.5 \mathrm{~km}$ and inside the outer solid curve to $5 \mathrm{~km}$ )

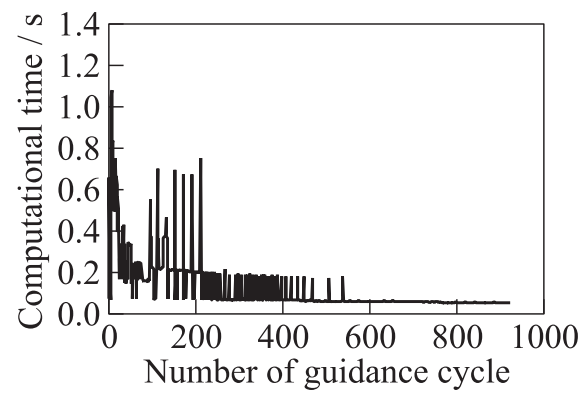

(a)

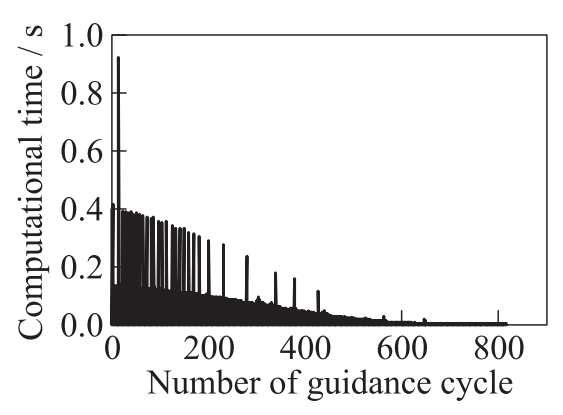

(b)

Figure 7 Computational time in one guidance cycle: $(a)$ in phases I, II, and III; and (b) in phase IV 
The corresponding altitude and steering profiles are given in Figs. $6 a$ and $6 b$. The flight path variables, including $g$-load factor are shown in Fig. $6 c$.

Figure 7 shows the time property of the guidance algorithm for a random trajectory in the Monte-Carlo simulation in which the guidance cycle is set as $2 \mathrm{~s}$. Figure $7 a$ gives the computation time in every guidance cycle in phases I, II, and III. Every time expense is less than $2 \mathrm{~s}$. Figure $7 b$ gives the computation time in every guidance cycle in phase IV which is also less than the guidance cycle.

\section{CONCLUDING REMARKS}

This paper has presented a new predictor-corrector skip entry guidance for vehicles with low lift-to-drag $(L / D)$ ratios which will be potentially used in the near-future Moon exploration mission with next generation onboard computer processor. The guidance scheme includes a trajectory planning algorithm and a closed-loop guidance law executed in each guidance cycle during skip entry. The numerical predictor-corrector method is used to compute the parameterized bank angle to make the trajectory satisfy the downrange accuracy while the bank reversal logic is utilized to make sure the entry vehicle guided to the landing site precisely. The standard condition and the Monte-Carlo simulations of the proposed guidance law are carried out. It demonstrates that the guidance law is valid in the varied reentry situation in lunar return missions, including direct entry, loft entry, and skip entry cases. And the robustness of the algorithm is also evaluated through simulations considering dispersions on the reentry initial states, the air density, the mass of vehicle, and the aerodynamic coefficients.

\section{REFERENCES}

1. Jeremy, R. R., and R. R. Zachry. 2007. A comparison of two Orion skip entry guidance algorithms. AIAA Guidance, Navigation and Control Conference and Exhibit. $19 \mathrm{p}$.

2. Bairstow, S.H., and G.H. Barton. 2007. Orion reentry guidance with extended range capability using PredGuid. AIAA Guidance, Navigation and Control Conference and Exhibit. 17 p.

3. Putnam, Z. R., S. H. Bairstow, R. D. Braun, et al. 2008. Improving lunar return entry range capability using enhanced skip trajectory guidance. J. Spacecraft Rockets 45:309-315.

4. Christopher, W. B., and L. Ping. 2008. Skip entry trajectory planning and guidance. J. Guid. Control Dynam. 31:1210-1219. 
5. Christopher, W.B., and L. Ping. 2010. Comparison of numerical predictorcorrector and Apollo skip entry guidance algorithms. AIAA Guidance, Navigation, and Control Conference. $20 \mathrm{p}$.

6. Vernis, P., F. Spreng, and G. Gelly. 2011. Accurate skip-entry guidance for low to medium $L / D$ spacecrafts return missions requiring high range capabilities. AIAA Guidance, Navigation, and Control Conference. 15 p.

7. Ping, L. 2008. Predictor-corrector entry guidance for low-lifting vehicles. J. Guid. Control Dynam. 31:1067-1075.

8. Moseley, P.E. 1969. The Apollo entry guidance: A review of the mathematical development and its operational characteristics. Huston, TX. TRW 69-FMT-791.

9. De Nicola, D., and V. Antonio. 2010. Fully structured aerodynamic model for parameter identification of a reentry experimental vehicle. J. Spacecraft Rockets 47:113-124.

10. Gamble, J. D., C. J. Cerimele, T.E. Moore, and J. Higgins. 1988. Atmospheric guidance concepts for an aeroassisted flight experiment. J. Astronaut. Sci. 36:4571.

11. Powell, R. W., and R. D. Braun. 1993. Six-degree-of-freedom guidance and control analysis of Mars aerocapture. J. Guid. Control Dynam. 16:1038-1044.

12. Evans, S. W., and G. A. Dukeman. 1995. Examination of a practical aerobraking guidance algorithm. J. Guid. Control Dynam. 18:471-477.

13. Bairstow, S.H. 2006. Reentry guidance with extended range capability for low $L / D$ spacecraft. Cambridge, MA: Department of Aeronautics and Astronautics, Massachusetts Institute of Technology. M.S. Thesis.

14. Zarchan, P., and H. Musoff. 2005. Fading memory filter. Ch. 15. Fundamentals of Kalman filtering: A practical approach. 2nd ed. Progress in astronautics and aeronautics ser. Reston, VA: AIAA. 208:647-676. 